\title{
Dental and Dental Hygiene Students' Perceptions of a Standardized Patient Instructor Conflict Resolution Program
}

\author{
Bridget E. Beattie, M.S.D.H.; Janet Kinney, R.D.H., M.S.; Mark Fitzgerald, D.D.S., M.S.; \\ Carol Anne Murdoch-Kinch, D.D.S., Ph.D.; Marilyn K. Guenther, M.S.; Karen Ridley, \\ R.D.H., M.S.; Laurie Whitman, M.S.E.; Vidya Ramaswamy, Ph.D.
}

Abstract: The aim of this study was to examine students' perceptions of the value of a standardized patient instructor conflict resolution program designed to strengthen their communication and confidence during difficult patient conversations. Three cohorts of students at one dental school were part of the study: the dental class of 2013, the dental class of 2014, and the dental hygiene class of 2013. The same groups of students completed surveys immediately following the program and one, two, or three years afterwards. Response rates for the survey immediately after the program were 98 percent $(n=99)$ of the dental class of 2013, 97 percent $(n=103)$ of the dental class of 2014, and 100 percent $(n=25)$ of the dental hygiene class of 2013. Response rates for the subsequent survey were 41.5 percent $(n=42)$ of the dental class of $2013,74.5$ percent $(n=79)$ of the dental class of 2014 , and 100 percent $(n=25)$ of the dental hygiene class of 2013. In the results, all students reported a high level of satisfaction in their immediate assessment of the program and its ability to prepare them for conflict situations. They also reported a high level of satisfaction in their retrospective self-assessment of conflict resolution skills. However, their assessment of the program's value and applicability appeared to have diminished over time. This study suggests that the program should continue being a part of both dental and dental hygiene curricula, with more training and guided experiences in self-assessment and perhaps supplemental experiences added.

\begin{abstract}
Ms. Beattie is Adjunct Clinical Lecturer of Dental Hygiene, Department of Periodontics and Oral Medicine, University of Michigan School of Dentistry; Ms. Kinney is Director of Dental Hygiene, Department of Periodontics and Oral Medicine, University of Michigan School of Dentistry; Dr. Fitzgerald is Associate Chair, Department of Cariology, Restorative Sciences, and Endodontics, University of Michigan School of Dentistry; Dr. Murdoch-Kinch is Associate Dean for Academic Affairs, University of Michigan School of Dentistry; Ms. Guenther is former Head of Standardized Patient Program, University of Michigan School of Dentistry; Ms. Ridley is Assistant Professor of Dental Hygiene, Department of Periodontics and Oral Medicine, University of Michigan School of Dentistry; Ms. Whitman is Training Specialist, University of Michigan; and Dr. Ramaswamy is Curriculum Assessment Specialist, University of Michigan School of Dentistry. Direct correspondence and requests for reprints to Dr. Vidya Ramaswamy, University of Michigan, School of Dentistry, 1011 N. University Avenue, Room 1204, Ann Arbor, MI 48109-1078; 734-647-4218; ramaswav@umich.edu.
\end{abstract}

Keywords: dental education, dental hygiene education, communication, conflict resolution, standardized patients

Submitted for publication 9/30/13; accepted 2/5/14

1 standardized patient is a trained actor who takes on characteristics of real patients in order to provide a learning opportunity for students. ${ }^{1}$ Dr. Howard Barrows at the University of Southern California introduced the use of standardized patients (SPs) in health professions education in $1963 .^{2}$ His use of these "programmed patients" was designed to help medical, nursing, and dental schools increase their students' success in clinical communications.

Since their introduction, SP programs have been utilized in health professions schools across the United States with the goal of improving clinicianpatient interactions. SPs are often chosen to portray patients with conditions that mirror their own medical histories in order to be perceived as authentic by the student clinician. ${ }^{3}$ The majority of research conducted on SP programs has been in medical education, with one study finding an increase in medical students' confidence and validation of their knowledge and critical thinking skills. ${ }^{4}$ The aim of our study was to investigate the perceived value of a similar educational intervention in dental education. The research obtained from this study will provide information on the program's strengths and weaknesses to facilitate future improvement and may aid other dental schools in implementing similar programs.

\section{Standardized Patient Instructor Program}

In 2001, the University of Michigan School of Dentistry expanded on the SP concept to create a 
standardized patient instructor (SPI) program. The addition of "instructor" to the position means this person is not only an actor who portrays patients but also serves as an advocate for patients and provides students with immediate feedback. The program was designed in part to facilitate interaction of dental and dental hygiene students with patients in a controlled setting, and it runs across all years of both dental and dental hygiene curricula, focusing on health history review, patient education, and treatment plan negotiations. The SPI method of providing instant feedback gives students the opportunity to refine their listening and communication skills when interacting with patients. Active, reflective, and empathic listening skills are acknowledged, critiqued, and reaffirmed by the SPI. These simulations are also used to teach students the importance of communication skills and the connection between communication and clinical reasoning necessary for optimal patient care. ${ }^{5}$

The majority of SPIs who work at the dental school have a health care background and utilize this knowledge, along with additional training, to present a realistic and convincing patient scenario. The SPIs are temporary, part-time employees and are paid approximately $\$ 25$ per hour for their work and $\$ 15$ per hour for training. They have been trained in three key tasks for the performance of their role: portrayal of a specific set of behaviors pertinent to the case scenario, assessment of students' clinical and communication skills pertinent to the scenario, and provision of written and oral feedback to the students. Each case scenario usually requires a fourhour training session for the SPIs accompanied by approximately three hours of homework. However, cross-training of SPIs across scenarios adds to their ability to help students improve their patient-centered communication skills. SPIs also develop a broader perspective as they mature through opportunities in their program. Their duties depend on the case.

In 2006, a conflict resolution (CR) component was added to the SPI program to further strengthen students' communications and confidence during difficult patient conversations. The addition was prompted by two goals: preparing students to function effectively with patients as they enter the clinical phase of their education, and preparing students to deal with ethical dilemmas in real time during patient interactions. In the CR scenario, students move in groups of three to three SPIs portraying different patient challenges. At each thirty-minute patient encounter, a different student assumes the role of provider while the other two become active observers, completing a written assessment and providing oral feedback after the interaction. To prepare students for the set of three patient encounters, they attend one lecture in conflict resolution and complete a mandatory preparation assignment. For the CR session, SPIs are trained to portray either an angry patient or a patient in pain and are given the guideline "Performance Keys for Listening and Interviewing Skills" to provide the students with guidance and feedback following the experience.

After six years, the CR component has become an integral part of this school's SPI program and is mandatory for all students as part of both dental and dental hygiene curricula. However, neither its effectiveness in teaching communication skills and enhancing student confidence nor the students' perceived value of such skills had previously been assessed. The aim of this study was to assess the program's effectiveness and students' perceptions of it.

\section{Methods}

This study was approved by the University of Michigan's Health Sciences and Behavioral Sciences Institutional Review Board, which deemed the surveys exempt on October 15, 2012. Our examination of students' perceptions of their SPI-CR experience collected data from two sources. The first data set was from surveys completed by students immediately following their SPI-CR experience. The second data set was from surveys completed by the same students one, two, or three years after the SPI-CR experience occurred. The study had three goals. One was to describe the immediate student evaluations of the SPI-CR experience using the Immediate Value Measure (IVM). A second goal was to describe their evaluations of the SPI-CR component one, two, or three years after the experience using the Perceived Retrospective Value Measure (PRVM). The final goal was to evaluate the psychometric properties of the PRVM, which was piloted in this study.

Three cohorts of students (dental class of 2013, dental class of 2014, and dental hygiene class of 2013) took part in the study. These dental and dental hygiene students participated in the SPI-CR experience and subsequently completed the immediate evaluations using the IVM measure. These data were accessed by the student investigator in fall 2012, when all students completed a retrospective evaluation of the SPI-CR training using the PRVM one, two, or three years after the SPI-CR experience. 
The first survey, the IVM, was developed based on similar surveys previously used in the university's medical and dental schools. It consisted of four statements ranked on a five-point Likert scale from $1=$ strongly disagree to $5=$ strongly agree and reflected students' immediate evaluations of their SPI-CR experience with respect to improving communication skills and increasing confidence during difficult patient conversations. Students used the principles of CR to address three scenarios, each lasting five to ten minutes (all materials are available from the corresponding author). Students completed the IVM in approximately ten minutes after conclusion of the SPI-CR session. The IVM has been used for both dental and dental hygiene students since 2006 with the implementation of the SPI-CR program.

The second survey, the PRVM, examined student perceptions of the continued perceived value and self-reported application of skills learned in the SPI-CR component one, two, and three years after the SPI-CR experience. This pilot survey consisted of eleven statements ranked on a five-point Likert scale ranging from $1=$ strongly disagree to $5=$ strongly agree. The survey was separated into two dimensions to measure both perceived skill retention and program value. The committee developing the survey justified each survey item developed (Table 1). The first question asked if students had received additional CR training since their participation in the SPI program that might affect their responses and attitudes towards the CR component. Questions 2 through 11 made up the actual measure. The second question asked students whether or not the SPI-CR scenarios were realistic. Questions three through six referenced individual goals of the program, asking if students perceived they emphasized collaboration, allowed for complete disclosure, looked for win-win outcomes, and emphasized mutual respect during the SPI-CR session. The seventh question inquired about students' use of listening skills, and the eighth asked if the session contributed to their ability to handle conflict situations both in and out of clinic. The ninth and tenth questions asked about students' value of the SPI-CR experience and if it should be continued. The final question asked if students would be interested in receiving additional $\mathrm{CR}$ training. All data analysis

Table 1. Rationale for development of statements in the Perceived Retrospective Value Measure (PRVM)

PRVM Statement

1. I have received additional conflict resolution training since my initial SPI-CR session.

2. Based on my experience with patients, the three SPI-CR scenarios were realistic.

3. During conflict, I emphasize collaboration and take common ownership of the problem.

4. During conflict, I allow for complete disclosure by all parties.

5. During conflict, I look for "win-win" outcomes.

6. During conflict, I emphasize mutual respect and responsibility.

7. During conflict, I use listening skills such as paraphrasing, reflecting, and summarizing.

8. The SPI-CR session contributed to my ability to handle conflictive situations both in and out of clinic.

9. The SPI-CR session was a valuable experience.

10. I would recommend the SPI-CR session be continued.

11. I would be interested in additional conflict resolution training.

$\begin{array}{cc} & \text { Skill (S) or } \\ \text { Rationale } & \text { Value (V) }\end{array}$

Additional training may affect their responses and attitude towards the conflict resolution component.

To measure the perceived usefulness of using actors as patients.

To measure the perceived success of this individual goal of the SPI program.

To measure the perceived success of this individual goal of the SPI program.

To measure the perceived success of this individual goal of the SPI program.

NA

V

S

S

S

To measure the perceived success of this individual goal $\mathrm{S}$ of the SPI program.

To measure the perceived success of this individual goal $\mathrm{S}$ of the SPI program.

To measure the perceived ability of this program to enhance conflict resolution skills.

To discover if students find this component of the SPI program relevant.

As graduates of this program, they have first-hand knowledge of its applicability.

To discover if time spent on this component was sufficient or not.

SPI-CR=standardized patient-instructor conflict resolution 
was performed using the Statistical Package for the Social Sciences (SPSS).

\section{Results}

Response rates for the IVM were as follows: 98 percent ( $\mathrm{n}=99$ ) of the dental class of 2013, 97 percent $(n=103)$ of the dental class of 2014, and 100 percent $(n=25)$ of the dental hygiene class of 2013 (Table 2). On this survey, taken immediately after the SPI-CR program, high percentages of students (92 percent to 100 percent) in all classes agreed/strongly agreed with the four statements about the usefulness of the motivational interviewing techniques (Figure 1).

Response rates for the PRVM were as follows: 41.5 percent $(n=42)$ of the dental class of $2013,74.5$ percent $(n=79)$ of the dental class of 2014, and 100 percent $(n=25)$ of the dental hygiene class of 2013
(Table 1). The lower response rate for the dental class of 2013 was possibly due to the high number of rotations outside the school students have in the fourth year. An independent samples t-test analysis was used to determine whether having additional CR training since the SPI-CR session had a significant effect on the responses to questions 3-8, which measured students' self-evaluation of their conflict resolution skills. Additional CR training did not have a statistically significant effect, so question 1 was not controlled for during the comparison of the groups $(\mathrm{p}=0.976)$.

A high percentage of students ( 75 percent to 100 percent) in all classes agreed/strongly agreed with the five statements asking about the skills aspect of the SPI-CR experience (statements 3 through 7, Table 3). In other words, students perceived the SPI-CR training as having a positive impact on

Table 2. Number of respondents and response rates to two surveys: Immediate Value Measure and Perceived Retrospective Value Measure

\begin{tabular}{lcccc}
\multirow{2}{*}{ Cohort } & \multicolumn{2}{c}{ Immediate Value Measure } & \multicolumn{2}{c}{ Perceived Retrospective Value Measure } \\
& When Completed & Response Rate & When Completed & Response Rate \\
\hline Dental class of 2013 & Fall 2009/D1 & $99(98 \%)$ & Fall 2012/D4 & $42(41.5 \%)$ \\
Dental class of 2014 & Fall 2010/D1 & $103(97 \%)$ & Fall 2012/D3 & $79(74.5 \%)$ \\
Dental hygiene class of 2013 & Winter 2012/DH3 & $25(100 \%)$ & Fall 2012/DH4 & $25(100 \%)$
\end{tabular}

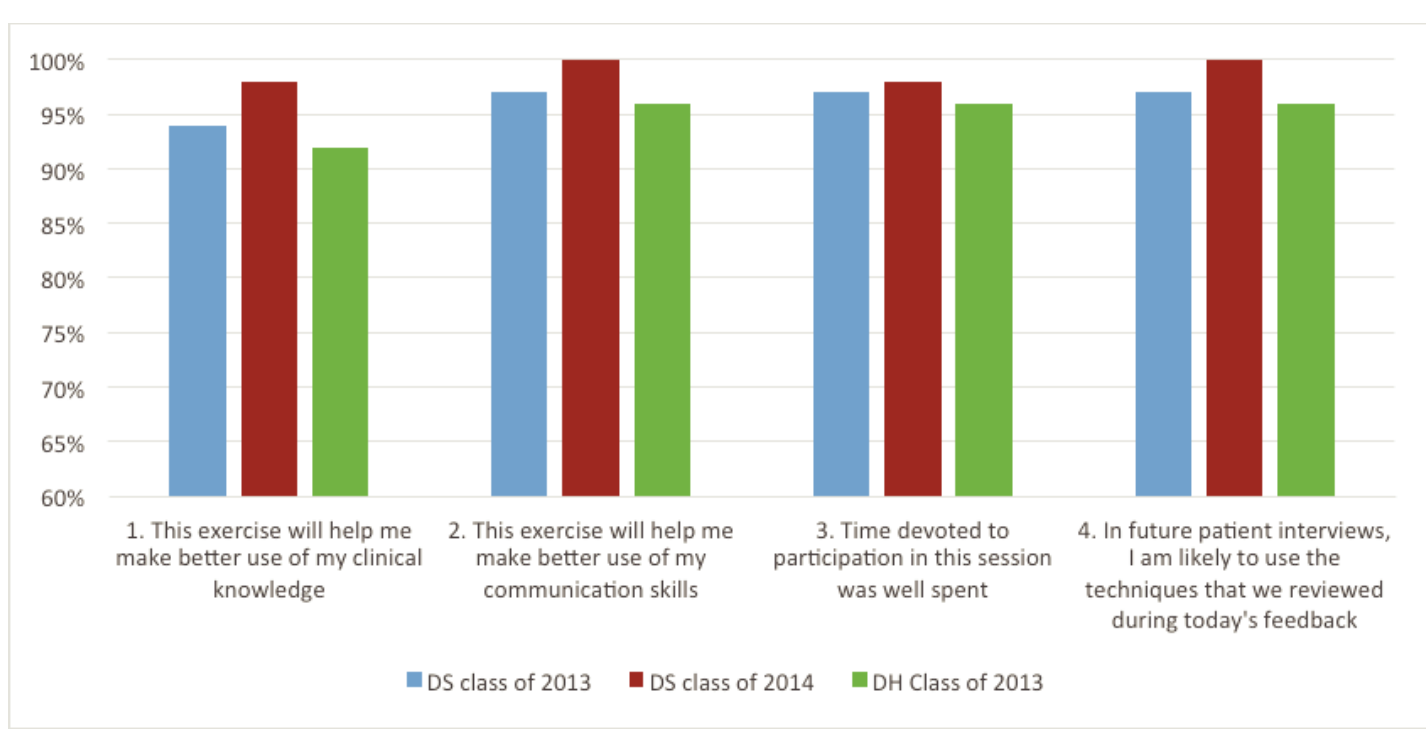

Figure 1. Percentage of students in dental (DS) and dental hygiene (DH) classes who agreed/strongly agreed with each statement on the Immediate Value Measure

Note: Y-axis scale goes from 60 to 100 percent. 
their skill development such as emphasizing collaboration and mutual respect, allowing for complete disclosure, using listening skills, and looking for "win-win" outcomes. A lower percentage of students (28 percent to 79 percent) in all classes agreed with the five statements (statement 2 and statements 8 through 11) asking about the "value" of the SPI-CR component.

Exploratory factor analysis (EFA) was used to examine the internal structure of the measure. The EFA was conducted using principal component analysis (with Varimax rotation) with Eigenvalues set at 1.5. The EFA showed two distinct factor loading structures (Table 4). One factor was named the "value" factor as items loading high on this factor were related to the perceived value of the SPI-CR experience. The second factor was named the "skill" factor as items loading high on this factor were about what skills were used during the conflict situation. Item total correlations were all significant ranging from 0.38 to 0.75 . The two factors did not correlate significantly with each other, but correlated significantly with total score (Table 5) and accounted for 56 percent of variance in scores together.

Table 3. Students who agreed/strongly agreed with statements $\mathbf{2}$ through $\mathbf{1 1}$ in the Perceived Retrospective Value Measure, by number and percentage of each class

Dental Class Dental Class Dental Hygiene of $2013 \quad$ of $2014 \quad$ Class of 2013

\section{Value Subscale}

2. Based on my experience with patients, the three SPI-CR scenarios were realistic.

8. The SPI-CR session contributed to my ability to handle conflictive situations both in and out of clinic.

9. The SPI-CR session was a valuable experience.

10. I would recommend the SPI-CR session be continued.

11. I would be interested in additional conflict resolution training.

$\begin{array}{lcc}18(43 \%) & 50(63 \%) & 19(76 \%) \\ 22(52 \%) & 46(58 \%) & 15(60 \%) \\ 24(57 \%) & 56(71 \%) & 11(44 \%) \\ 27(64 \%) & 62(79 \%) & 12(48 \%) \\ 20(48 \%) & 26(33 \%) & 7(28 \%) \\ & & \\ 34(81 \%) & 60(76 \%) & 22(88 \%) \\ 32(76 \%) & 59(75 \%) & 21(84 \%) \\ 38(90 \%) & 69(87 \%) & 23(92 \%) \\ 36(86 \%) & 76(96 \%) & 25(100 \%) \\ 37(88 \%) & 67(85 \%) & 22(88 \%)\end{array}$

\section{Skills Subscale}

3. During conflict, I emphasize collaboration and take common ownership of the problem.

4. During conflict, I allow for complete disclosure by all parties.

5. During conflict, I look for "win-win" outcomes.

6. During conflict, I emphasize mutual respect and responsibility.

7. During conflict, I use listening skills such as paraphrasing, reflecting, and summarizing.

\section{Table 4. Exploratory factor analysis using Varimax rotation and item-total correlation}

\begin{tabular}{|c|c|c|c|c|}
\hline $\begin{array}{l}\text { Item } \\
\text { No. }\end{array}$ & Item & $\begin{array}{l}\text { Factor One: } \\
\quad \text { Value }\end{array}$ & $\begin{array}{l}\text { Factor Two: } \\
\quad \text { Skill }\end{array}$ & $\begin{array}{l}\text { Item-Total } \\
\text { Correlation }\end{array}$ \\
\hline 2 & Based on my experience with patients, the three SPI-CR scenarios were realistic. & 0.66 & 0.17 & $0.61 *$ \\
\hline 8 & $\begin{array}{l}\text { The SPI-CR session contributed to my ability to handle conflictive situations } \\
\text { both in and out of clinic. }\end{array}$ & 0.79 & 0.16 & $0.71 *$ \\
\hline 9 & The SPI-CR session was a valuable experience. & 0.91 & -0.03 & $0.72 *$ \\
\hline 10 & I would recommend the SPI-CR session be continued. & 0.89 & 0.05 & $0.75^{*}$ \\
\hline 11 & I would be interested in additional conflict resolution training. & 0.63 & -0.09 & $0.55^{*}$ \\
\hline 3 & $\begin{array}{l}\text { During conflict, I emphasize collaboration and take common ownership of } \\
\text { the problem. }\end{array}$ & 0.11 & 0.65 & $0.40^{*}$ \\
\hline 4 & During conflict, I allow for complete disclosure by all parties. & -0.01 & 0.72 & $0.40^{*}$ \\
\hline 5 & During conflict, I look for "win-win" outcomes. & -0.02 & 0.78 & $0.41^{*}$ \\
\hline 6 & During conflict, I emphasize mutual respect and responsibility. & 0.04 & 0.62 & $0.38^{*}$ \\
\hline 7 & $\begin{array}{l}\text { During conflict, I use listening skills such as paraphrasing, reflecting, and } \\
\text { summarizing. }\end{array}$ & 0.10 & 0.68 & $0.46^{*}$ \\
\hline
\end{tabular}

*Correlation significant at 0.01 level. 
Table 5. Pearson's product-moment correlations for factor one (value), factor two (skill), and total scale

\begin{tabular}{lccc} 
& Value & Skill & Scale Total \\
\hline Value & 1.00 & 0.15 & $0.87^{*}$ \\
Skill & & 1.00 & $0.62^{*}$
\end{tabular}

*Correlation significant at 0.01 level.

Table 6 shows the correlation between all the individual items. The descriptive statistics for each of the subscales and the total scale are shown in Table 7. Internal consistency reliability was calculated using Cronbach's $\alpha$ coefficient. The total scale and each of the subscales comprised of items making up the value factor and skill factor, respectively, had good reliability.

\section{Discussion}

The first goal of this study was to analyze student perceptions of the SPI-CR component to determine its immediate value to students. As previous studies have shown, ${ }^{6-9}$ the study aligns with overall positive responses from dental and dental hygiene students immediately following the SPI-CR training. First impressions of the SPI-CR program's ability to contribute to student communication skills and clinical knowledge were positively reported by 90 percent of those who participated. Other studies agree, showing that students' communication skills increased following participation in an SP experience..$^{3,10,11}$ The majority of the dental students in our study agreed that time devoted to participation in the CR session was well spent and correspondingly agreed it was a valuable session. In a study by Klamen and Yudkowsky, students also reported that participation in an SP session was valuable and a good use of time. ${ }^{7} \mathrm{~A}$ high percentage of dental and dental hygiene students in our study also agreed that they were likely to use the $\mathrm{CR}$ techniques reviewed during their feedback session with the SPIs (Figure 1).

The second goal of this study was to analyze the same students' perceptions of the school's SPI-CR component to determine the long-term retention of skills learned in this intervention. Results revealed that dental and dental hygiene students applied all of the CR techniques over time based on their selfassessment responses. However, the PRVM measure only consisted of a student self-assessment. The ability to successfully perform the CR skills over time is unknown without an external evaluation of their skills. Students might think they are doing a better job using the techniques than perhaps in reality they are. Sedikides states that individuals involved in

Table 6. Pearson's product moment correlations for all items on scale

\begin{tabular}{|c|c|c|c|c|c|c|c|c|c|c|}
\hline Item & 2 & 3 & 4 & 5 & 6 & 7 & 8 & 9 & 10 & 11 \\
\hline 2 & 1.00 & $0.20^{*}$ & 0.06 & 0.10 & 0.15 & 0.09 & $0.48^{* *}$ & $0.51^{* *}$ & $0.48^{* *}$ & $0.22 *$ \\
\hline 3 & & 1.00 & $0.46^{* *}$ & $0.32^{* *}$ & 0.15 & $0.30^{* *}$ & 0.15 & 0.06 & 0.07 & 0.07 \\
\hline 4 & & & 1.00 & $0.48^{* *}$ & $0.26^{* *}$ & $0.31^{* *}$ & 0.04 & 0.03 & 0.05 & 0.05 \\
\hline 5 & & & & 1.00 & $0.41^{* *}$ & $0.40^{* *}$ & 0.14 & 0.00 & 0.04 & -0.07 \\
\hline 6 & & & & & 1.00 & $0.38^{* *}$ & 0.13 & 0.03 & 0.06 & -0.02 \\
\hline 7 & & & & & & 1.00 & 0.19* & 0.02 & 0.15 & 0.06 \\
\hline 8 & & & & & & & 1.00 & $0.62^{* *}$ & $0.65^{* *}$ & $0.34^{* *}$ \\
\hline 9 & & & & & & & & 1.00 & $0.82^{* *}$ & $0.49^{* *}$ \\
\hline 10 & & & & & & & & & 1.00 & $0.45^{* *}$ \\
\hline 11 & & & & & & & & & & 1.00 \\
\hline
\end{tabular}

*Correlation significant at 0.05 level.

**Correlation significant at 0.01 level.

Note: Bold indicates significant correlation.

Table 7. Descriptive summary scores and Cronbach's $\alpha$ for scale $(n=146)$

\begin{tabular}{lcccccc} 
& No. of Items & Min & Max & Mean & SD & Cronbach's $\alpha$ \\
\hline Factor one: value & 5 & 2.00 & 24.00 & 12.45 & 3.56 & 0.84 \\
Factor two: skill & 5 & 5.00 & 16.00 & 9.75 & 2.23 & 0.73 \\
Total scale & 10 & 10.00 & 39.00 & 22.21 & 4.48 & 0.76 \\
\hline
\end{tabular}


self-assessment often enhance their own successes and downplay their failures because they cannot be impartial. ${ }^{12}$ The results seem to show this tendency. As the SPI program director for ten years, Marilyn Guenther reports seeing a pattern whereby as students succeed in the academic aspects of their education, they often hold a heightened perception of their self-assessment skills; that is, success in one area translates to success in another area. In her opinion, accurate self-assessment is a skill that must be taught, practiced, and repeatedly reinforced throughout the dental curriculum. While the dental and dental hygiene students have opportunities to self-assess, perhaps additional training and guided practice would lead to a lower self-assessment of their CR skills.

Another thought-provoking outcome of the study relating to the second goal was the finding that as dental and dental hygiene students gained more patient experience, their perceived value of the SPI-CR program slightly diminished. One would conjecture that the findings would be in agreement with those reported by Klamen and Yudkowsky, who found that six months following an SPI experience, students valued the experience and believed it helped prepare them for "real" patient care. ${ }^{7}$ However, our study found the opposite. Dental and dental hygiene students did not retain a constant level of perceived value for the SPI-CR program over time. The SPICR experience falls somewhat early in the students' educational programs; therefore, students may forget the importance of the experience over time. A possible solution would be integrating additional SPI-CR experiences, possibly through an objective structured clinical examination, throughout the dental and dental hygiene programs to reinforce its value and provide students with additional opportunities to enhance their CR skills.

A third goal was to evaluate the psychometric properties of the PRVM measure. Exploratory factor analysis suggests the tool measured a skills factor and a value factor. The skills subscale referred to the impact of the SPI-CR training on the use of skills such as abilities to collaborate and listen that are needed during an actual SPI-CR session. The value subscale asked students to evaluate the value of the SPI-CR experience. The identification of these two factors will help provide clarity in future studies that explore the impact of the SPI-CR experience with respect to one or both of the components. The reliability of the scale was excellent for the total scale as well as its subscales. Thus, this scale demonstrated good reli- ability and validity in a diverse sample of dental and dental hygiene students.

The primary strength of the SPI-CR program is the opportunity it provided students to learn the skills needed to interact effectively with patients during challenging situations. These experiences aid students in the development of professional conduct and support both the American Dental Association (ADA) and American Dental Hygienists' Association (ADHA) codes of ethics, which call for their members to follow high levels of ethical consciousness, decision making, and practice. ${ }^{13,14}$ However, this study is not without limitations. Certainly, having more participants in the study would have increased confidence in the generalizability of the results. This was the first time the CR component of the SPI program was assessed, opening future opportunities for the school to evaluate the effectiveness of the learning interventions offered by the program. Surveying graduates at various time periods after graduation to inquire about their perceptions of the value of the SPI-CR program and retention and use of $\mathrm{CR}$ techniques would be very interesting and would offer a different, perhaps more long-term view of the program. Furthermore, the selected research methodologies used in this study appear to be appropriate. However, there could be other evaluation measures better able to provide evidence-based outcomes. Having acknowledged these constraints, we believe the results bring worthwhile information to dental and dental hygiene education.

\section{Conclusion}

This study found the SPI-CR program was immediately valued by dental and dental hygiene students using the IVM measure, which had both reliability and validity. The students' self-assessment of their CR skills remained high one to three years following their initial SPI-CR experience. However, the students' perceptions of the SPI-CR program's value decreased as they advanced in their programs. In order to increase the validity of the students' self-assessments, we recommend that a training component in self-assessment be offered preceding or concurrently with the SPI-CR session. The SPI-CR program should be integrated more often into the dental and dental hygiene programs to reinforce both its importance and the students' CR skill development. 


\section{REFERENCES}

1. Wallace P. Following the threads of an innovation: the history of standardized patients in medical education. Caduceus 1997;13(2):5-28.

2. Hardee J, Kasper I. From standardized patient to care actor: evolution of a teaching methodology. Perm J 2005;9(3):79-82.

3. Wagner J, Arteaga S, D'Ambrosio J, et al. A patientinstructor program to promote dental students' communication skills with diverse patients. J Dent Educ 2007;71(12):1554-60.

4. Decker S, Sportsman S, Puetz L, Billings L. The evolution of simulation and its contribution to competence. J Contin Educ Nurs 2008;39(2):74-9.

5. Windish DM, Price EG, Clever SL, et al. Teaching medical students the important connection between communication and clinical reasoning. J Gen Intern Med 2005;20(12):1108-13.

6. Robins LS, Alexander GL, Dicken LL, et al. The effect of a standardized patient instructor experience on students' anxiety and confidence levels performing the male genitorectal examination. Teach Learn Med 1997;9(4):264-9.
7. Klamen D, Yudkowsky R. Using standardized patients for formative feedback in an introduction to psychotherapy course. Acad Psychiatry 2002;26(3):168-72.

8. Anderson M, Holmes T, LeFlore J, et al. Standardized patients in educating student nurses: one school's experience. Clin Sim Nurs 2010;6:e61-e66.

9. Koerber A, Crawford J, O'Connell K. The effects of teaching dental students brief motivational interviewing for smoking-cessation counseling: a pilot study. J Dent Educ 2003;67(4):439-47.

10. Yedidia M, Gillespie C, Kachur E, et al. Effect of communications training on medical student performance. JAMA 2003;290(9):1157-65.

11. Broder H, Janal M. Promoting interpersonal skills and cultural sensitivity among dental students. J Dent Educ 2006;70(4):409-16.

12. Sedikides C. Assessment, enhancement, and verification determinants of the self-evaluation process. J Pers Soc Psychol 1993;65(2):317-38.

13. American Dental Association. ADA principles of ethics and code of professional conduct. Chicago: American Dental Association, 2012.

14. American Dental Hygienists' Association. ADHA bylaws and code of ethics. Chicago: American Dental Hygienists' Association, 2013. 